\title{
Nitriles in Heterocyclic Synthesis: New Approaches for Synthesis of Some Pyridine Derivatives
}

\author{
N. S. Ibrahim*,a, R. M. Mohareb ${ }^{\mathrm{a}}$, and H. Z. Shams ${ }^{\mathrm{b}}$ \\ Department of Chemistry, Faculty of Science, Cairo ${ }^{a}$ University, Giza, Egypt and \\ Chemistry Department, Faculty of Science, Helwan ${ }^{\mathrm{b}}$ University, Helwan, Egypt \\ Z. Naturforsch. 43b, 1351-1354 (1988); received March 31, 1987/June 16, 1988 \\ Cyanoethanoic Hydrazide, Heterocyclic Synthesis, Pyridine Derivatives
}

A variety of pyridines was prepared from reaction of $\mathrm{N}$-benzylidenecyanoethanoic hydrazide (2) with malononitrile, benzoylacetonitrile, cyanoacetamide and cinnamonitrile derivatives. The reactivity of $\mathbf{2}$ towards a variety of ketones was studied.

Polyfunctional nitriles are highly reactive reagents that have been extensively used in heterocyclic synthesis [1-3]. In continuation of our program directed to the development of new procedures for synthesis of azoles, azines, and their condensed derivatives from readily available materials $[4,5]$, we report here a new synthesis of substituted pyridines from polyfunctional nitriles. Reaction of cyanoethanoic hydrazide (1) with benzaldehyde affords a 1:1 condensation product. Structure 2 was established for the reaction product based on ${ }^{1} \mathrm{H}$ NMR spectrum which revealed a singlet at $\delta 4.0 \mathrm{ppm}$ for the methylene protons. A signal of only one $\mathrm{D}_{2} \mathrm{O}$ exchangeable proton at $\delta 5.5 \mathrm{ppm}$ was observed. If this product was 3, three $\mathrm{D}_{2} \mathrm{O}$ exchangeable protons should have been observed.

Compound 2 reacted with malononitrile to yield a 1:1 adduct. Structure 5 was assigned for the reaction product based on IR, ${ }^{1} \mathrm{H}$ NMR and mass spectra. IR spectrum revealed only one $\mathrm{CN}$ stretching band at $v=$ $2220 \mathrm{~cm}^{-1}$. The ${ }^{1} \mathrm{H}$ NMR spectrum revealed signals of four $\mathrm{D}_{2} \mathrm{O}$ exchangeable protons at $\delta 5.6$ and $8.3 \mathrm{ppm}$ corresponding to the two amino functions. A multiplet for aromatic and arylidene protons appeared at $\delta 7.2-7.6 \mathrm{ppm}$. Structure 5 was assumed to be formed through the intermediate 4 .

Compound 2 reacted with cyanoacetamide affording the pyridine derivative $\mathbf{6}$, through the cyclization of the non-isolable adduct 7. Structure 6 was established based on the IR spectrum which revealed the absence of $\mathrm{CN}$ stretching band expected at $\sim 2200 \mathrm{~cm}^{-1}$. Compound 2 condensed with benzoyl-

* Reprint requests to Dr. N. S. Ibrahim.

Verlag der Zeitschrift für Naturforschung, D-7400 Tübingen 0932-0776/88/1000-1351/\$01.00/0 acetonitrile to yield the $\mathrm{N}$-aminopyridine 8 . Compound $\mathbf{2}$ reacted with cinnamonitrile derivatives $9 \mathbf{a}, \mathbf{b}$ affording products of molecular formulae $\mathrm{C}_{20} \mathrm{H}_{13} \mathrm{~N}_{5} \mathrm{O}$ and $\mathrm{C}_{26} \mathrm{H}_{18} \mathrm{~N}_{4} \mathrm{O}_{2}$ respectively. Pyridine structure $\mathbf{1 2}$ was assigned based on IR and ${ }^{1} \mathrm{H}$ NMR data. The formation of $\mathbf{1 2}$ in this reaction was assumed to proceed via addition of the methylene group in $\mathbf{2}$ to the activated double bond in $\mathbf{9}$ to yield the acyclic intermediate 10 which cyclizes into 11. This is followed by aromatization via loss of hydrogen molecule to yield 12. Ready autoxidation of dihydropyridines into pyridines under similar conditions has been reported [6]. Compound 2 condensed with acetophenone and ethyl acetoacetate yielding $\mathbf{1 3}$ and $\mathbf{1 4}$. In case of acetylacetone, compound $\mathbf{2}$ underwent condensation reaction yielding the pyridine derivative $\mathbf{1 5}$ via loss of two water molecules.

Compound 14 coupled with benzenediazonium chloride to yield the hydrazone $\mathbf{1 7}$ which was converted into the pyridazin-6-imine (18) on refluxing in acetic-hydrochloric acid mixture. Structure $\mathbf{1 8}$ was established based on the IR spectrum which revealed the absence of $\mathrm{CN}$ stretching and expected at $\sim 2200 \mathrm{~cm}^{-1}$.

Compound $\mathbf{1 4}$ reacted with trichloroacetonitrile to yield the pyridine derivative $\mathbf{2 0}$ which is formed through cyclization of the non isolable intermediate $\mathbf{1 9}$ as reported [1].

\section{Experimental}

All melting point are uncorrected. IR spectra were recorded on a Pye-Unicam Spectrophotometer. ${ }^{1} \mathrm{H}$ NMR spectra on a Varian EM-90 $\mathrm{MHz}$ spectrometer. The Microanalytical data were performed by the Microanalytical Unit at Cairo University. 


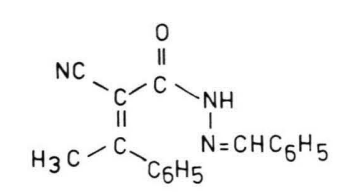

13

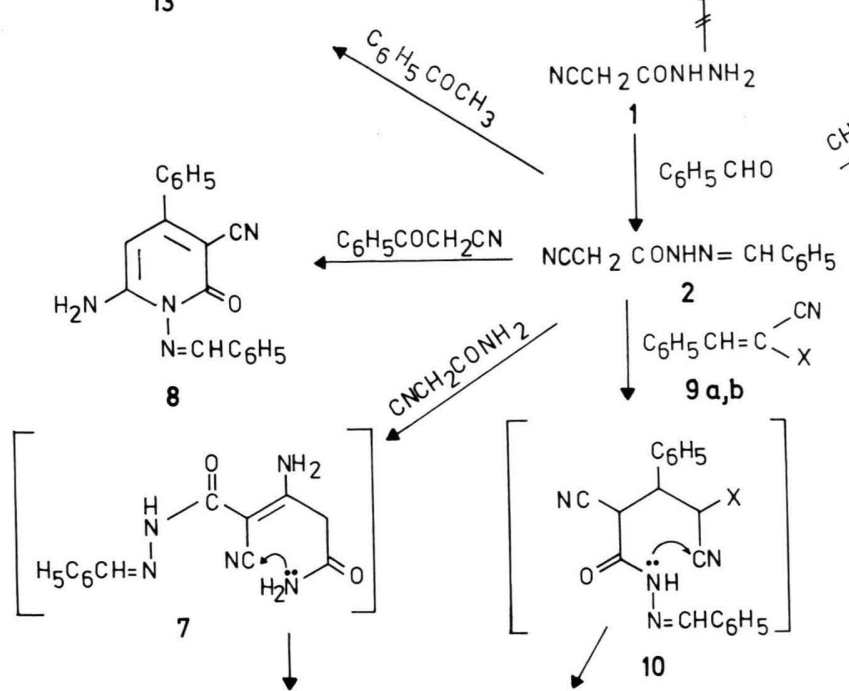

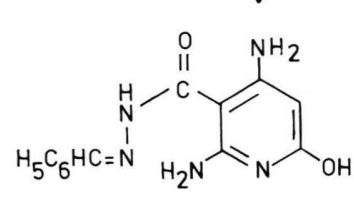

6

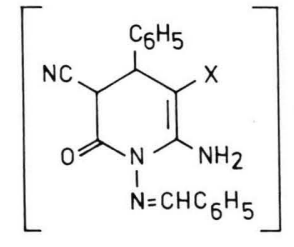

11<smiles>N#C/C(=C/c1ccccc1)C(=O)NN</smiles>

3

$\mathrm{H}_{3} \mathrm{C}^{-}-\mathrm{C}_{14} \mathrm{CH}_{2} \mathrm{CO}_{2} \mathrm{C}_{2} \mathrm{H}_{5}$
14
$\underbrace{\mathrm{NH}}_{\mathrm{N}=\mathrm{CHC}_{6} \mathrm{H}_{5}}$

5

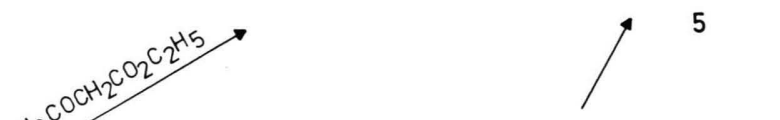

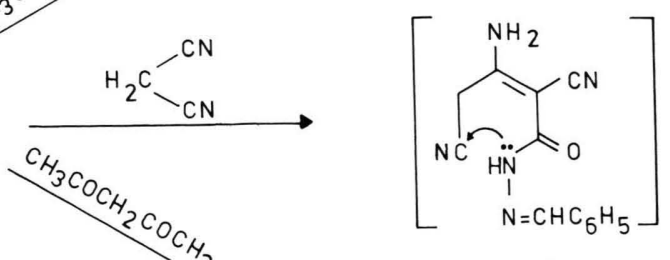

4<smiles>Cc1cc(C)n(/N=C\c2ccccc2)c(=O)c1C#N</smiles>

15<smiles></smiles>

$12 \mathrm{a}: \mathrm{X}=\mathrm{CN}$

12b: $X=\mathrm{COC}_{6} \mathrm{H}_{5}$

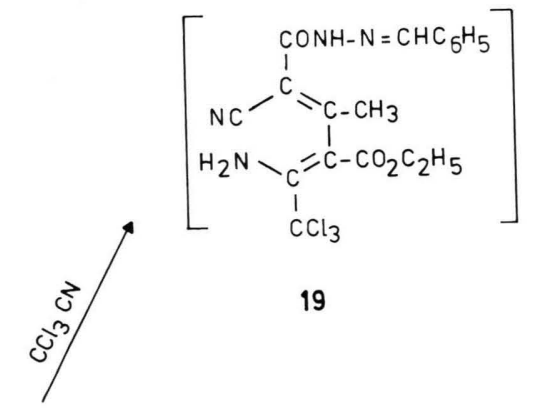

$\longrightarrow$

20

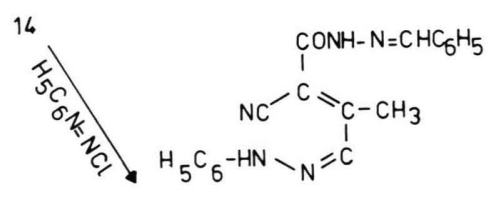

17

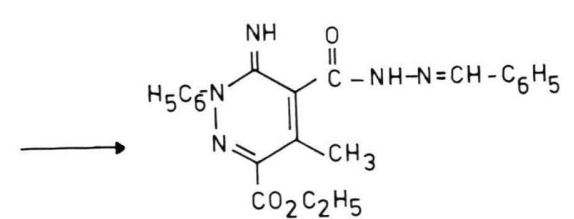

18 


\section{$N$-Benzylidenecyanoethanoic hydrazide (2)}

Compound 1 ( $0.99 \mathrm{~g}, 0.01$ mole $)$ was refluxed with ( $1.06 \mathrm{~g}, 0.01 \mathrm{~mole})$ of benzaldehyde in $20 \mathrm{ml}$ of ethanol catalyzed with piperidine for $2 \mathrm{~h}$. The solid product, so formed, was filtered off and crystallized from ethanol as colourless crystals; m.p. $165^{\circ} \mathrm{C}$, yield $1.77 \mathrm{~g}(95 \%)$. - IR $\left(\mathrm{KBr}, \mathrm{cm}^{-1}\right): v(\mathrm{NH}): 3300$, $v(\mathrm{CN}): 2200, v(\mathrm{CO}): 1700 .-{ }^{1} \mathrm{H}$ NMR (DMSO): $\delta=4.0 \mathrm{ppm}\left(\mathrm{s}, \mathrm{CH}_{2}\right) ; 5.5(\mathrm{~s}, \mathrm{NH}) ; 7.2-7.6(\mathrm{~m}$, $\left.\mathrm{C}_{6} \mathrm{H}_{5}, \mathrm{CH}\right)$.

$$
\mathrm{C}_{10} \mathrm{H}_{9} \mathrm{~N}_{3} \mathrm{O} \text { (187.22) }
$$

$$
\text { Calcd C 64.14 H } 4.85 \text { N 22.44, }
$$$$
\text { Found C 64.20 H } 4.80 \quad \text { N 22.30. }
$$

\section{1-Benzalimino-4,6-diamino-2(2 H)-pyridinone- 3-carbonitrile (5)}

Compound 2 (1.87 g; 0.01 mole) was refluxed with $(0.66 \mathrm{~g}, 0.01$ mole $)$ malononitrile in ethanol $(20 \mathrm{ml})$ catalyzed with piperidine for $2 \mathrm{~h}$. The resulting solid product was collected by filtration and crystallized from acetic acid as colourless crystals; m.p. $312^{\circ} \mathrm{C}$; yield $2.27 \mathrm{~g}(90 \%)$. - IR $\left(\mathrm{KBr}, \mathrm{cm}^{-1}\right): v\left(\mathrm{NH}_{2}\right)$ : $3410,3390,3240 ; v(\mathrm{CN}): 2220(\mathrm{CN}) ; v(\mathrm{CO}): 1690$. ${ }^{1} \mathrm{H}$ NMR (DMSO): $\delta=5.6 \mathrm{ppm}\left(\mathrm{s}, \mathrm{NH}_{2}\right) ; 7.2-7.6$ $\left(\mathrm{m}, \mathrm{C}_{6} \mathrm{H}_{5}, \mathrm{H}-5\right.$, exocyclic $\left.\mathrm{CH}\right) ; 8.3\left(\mathrm{~s}, \mathrm{NH}_{2}\right)$. MS: $m / z=253\left(\mathrm{M}^{+}\right)$.

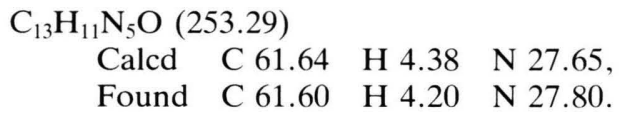

\section{5-Benzalhydrazido-4,6-diamino-2-hydroxypyridine (6)}

To a solution of compound 2 (1.87 g, 0.01 mole $)$ in dimethylformamide $(20 \mathrm{ml})$, cyanoacetamide ( $0.84 \mathrm{~g}, 0.01$ mole) was added. The reaction mixture was refluxed for $3 \mathrm{~h}$ then poured into ice/water containing few drops of hydrochloric acid. The solid product, so formed, was collected by filtration and crystallized from acetic acid as orange crystals; m.p. $250{ }^{\circ} \mathrm{C}$, yield $2.02 \mathrm{~g}(75 \%)$ - IR $\left(\mathrm{KBr}, \mathrm{cm}^{-1}\right)$ : $v(\mathrm{OH}): 3600 ; v\left(\mathrm{NH}_{2}, \mathrm{NH}\right): 3400,3200 ; v(\mathrm{CO}): 1710$.

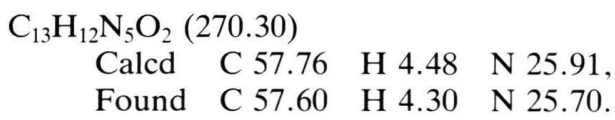

6-Amino-1-benzalimino-4-phenyl-2(2 H)-pyridinone3-carbonitrile (8)

Compound 2 (1.87 g, 0.01 mole) was refluxed with $(1.45 \mathrm{~g}, 0.01$ mole) benzoylacetonitrile in pyridine $(20 \mathrm{ml})$ for $2 \mathrm{~h}$. The reaction mixture was poured into water. The resulting solid product was collected by filtration and crystallized from DMF as brown crystals; m.p. $206{ }^{\circ} \mathrm{C}$; yield 2.04 g (65\%). - IR
$\left(\mathrm{KBr}, \mathrm{cm}^{-1}\right): v\left(\mathrm{NH}_{2}\right): 3400,3300 ; v(\mathrm{CN}): 2210$; $v(\mathrm{CO}): 1690 .-{ }^{1} \mathrm{H}$ NMR (DMSO): $\delta=3.0 \mathrm{ppm}(\mathrm{s}$, $\left.\mathrm{NH}_{2}\right) ; 7.4-7.9\left(\mathrm{~m}, 2 \times \mathrm{C}_{6} \mathrm{H}_{5}, \mathrm{H}-7\right.$, and exocyclic $\left.\mathrm{CH}\right)$.

$$
\begin{array}{rrrrr}
\mathrm{C}_{19} \mathrm{H}_{14} \mathrm{~N}_{4} \mathrm{O}(314.37) & & \\
\text { Calcd } & \mathrm{C} 72.58 & \mathrm{H} 4.49 & \mathrm{~N} 17.82, \\
\text { Found } & \mathrm{C} 72.60 & \mathrm{H} 4.50 & \mathrm{~N} 17.80 .
\end{array}
$$

\section{Reaction of $\mathbf{2}$ with cinnamonitrile derivatives $\mathbf{9 a , b}$}

Equimolecular amounts (0.01 mole) of either 9a $(1.54 \mathrm{~g})$ or $9 \mathbf{b}(2.33 \mathrm{~g})$ were refluxed with compound 2 ( $1.87 \mathrm{~g}, 0.01 \mathrm{~mole})$ in ethanol $(30 \mathrm{ml})$ catalyzed with piperidine for $5 \mathrm{~h}$. The solid products so formed, were collected by filtration and crystallized from the proper solvent.

\section{6-Amino-1-benzalimino-4-phenyl-2(2 H)pyridinone- 3,5-dicarbonitrile (12a)}

M.p. $302{ }^{\circ} \mathrm{C}$; yield $2.54 \mathrm{~g}(75 \%)$, buff crystals from DMF. - IR ( $\left.\mathrm{KBr}, \mathrm{cm}^{-1}\right): v\left(\mathrm{NH}_{2}\right): 3400,3300$; $v(\mathrm{CN}): 2210,2190 ; \quad v(\mathrm{CO}): 1700 . \quad-{ }^{1} \mathrm{H}$ NMR (DMSO): $\delta=3.3 \mathrm{ppm}\left(\mathrm{s}, \mathrm{NH}_{2}\right) ; 7.3-7.7(\mathrm{~m}$, $2 \times \mathrm{C}_{6} \mathrm{H}_{5}$, exocyclic $\mathrm{CH}$ ).

$$
\begin{array}{cccc}
\mathrm{C}_{20} \mathrm{H}_{13} \mathrm{~N}_{5} \mathrm{O}(339.38) & & \\
\text { Calcd } & \mathrm{C} 70.77 & \text { H } 3.86 & \text { N 20.64, } \\
\text { Found } & \text { C } 70.80 & \text { H } 3.80 & \text { N 20.50. }
\end{array}
$$

\section{6-Amino-1-benzalimino-5-benzoyl-4-phenyl- 2(2 H)-pyridinone-3-carbonitrile $(\mathbf{1 2 b})$}

M.p. $120{ }^{\circ} \mathrm{C}$; yield $2.50 \mathrm{~g}(60 \%)$; yellow crystals from ethanol. - IR $\left(\mathrm{KBr}, \mathrm{cm}^{-1}\right): v\left(\mathrm{NH}_{2}\right): 3400$, $3350 ; v(\mathrm{CN}): 2200 ; v(\mathrm{CO}): 1710,1690 .-{ }^{1} \mathrm{H}$ NMR (DMSO): $\delta=3.5 \mathrm{ppm}\left(\mathrm{s}, \mathrm{NH}_{2}\right) ; 7.3-7.9(\mathrm{~m}$, $3 \times \mathrm{C}_{6} \mathrm{H}_{5}$, exocyclic $\mathrm{CH}$ ).

$$
\begin{array}{rrrr}
\mathrm{C}_{26} \mathrm{H}_{18} \mathrm{~N}_{4} \mathrm{O}_{2}(418.48) & & \\
\text { Calcd } & \mathrm{C} 74.61 & \mathrm{H} 4.34 & \mathrm{~N} 13.39, \\
\text { Found } & \mathrm{C} 74.60 & \mathrm{H} 4.10 & \mathrm{~N} 13.50 .
\end{array}
$$

Reaction of $\mathbf{2}$ with acetophenone and ethyl acetoacetate to give 13 and 14 respectively

To a solution of compound 2 ( $1.87 \mathrm{~g}, 0.01$ mole $)$ in dimethylformamide $(20 \mathrm{ml})$ catalyzed with few drops of piperidine; either acetophenone (1.2 g, 0.01 mole) or ethyl acetoacetate $(1.3 \mathrm{~g}, 0.01$ mole $)$ was added. The reaction mixture was refluxed for $3 \mathrm{~h}$ and then poured into ice-water containing few drops of hydrochloric acid. The solid products, so formed, were collected by filtration and crystallized from the proper solvent.

\section{Compound 13}

M.p. $170{ }^{\circ} \mathrm{C}$; yield $2.31 \mathrm{~g}(80 \%)$; yellow crystals from ethanol. - IR (KBr, $\left.\mathrm{cm}^{-1}\right): v(\mathrm{NH}): 3300,3100$; 
$v(\mathrm{CN}): 2200 ; v(\mathrm{CO}): 1690 . \quad-{ }^{1} \mathrm{H}$ NMR (DMSO): $\delta=1.3 \mathrm{ppm}\left(\mathrm{s}, \mathrm{CH}_{3}\right) ; 7.2-7.9\left(\mathrm{~m}, 2 \times \mathrm{C}_{6} \mathrm{H}_{5}, \mathrm{CH}\right)$; 8.3 (br, NH).

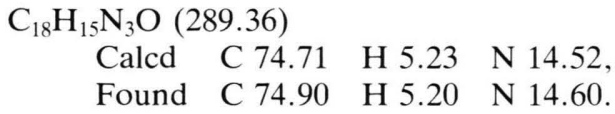

\section{Compound 14}

M.p. $250{ }^{\circ} \mathrm{C}$; yield $2.24 \mathrm{~g}(75 \%)$; orange crystals from acetic acid. - IR $\left(\mathrm{KBr}, \mathrm{cm}^{-1}\right): v(\mathrm{NH}): 3400$, $3350 ; \quad v(\mathrm{CN}): 2220 ; \quad v(\mathrm{CO}): 1710 . \quad-{ }^{1} \mathrm{H}$ NMR (DMSO): $\delta=1.2 \mathrm{ppm}\left(\mathrm{m}, 2 \times \mathrm{CH}_{3}\right) ; 4.2(\mathrm{~m}$, $\left.2 \times \mathrm{CH}_{2}\right) ; 7.2-7.7\left(\mathrm{~m}, \mathrm{C}_{6} \mathrm{H}_{5}, \mathrm{CH}, \mathrm{NH}\right)$.

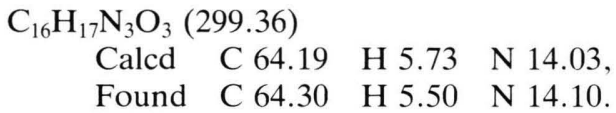

\section{1-Benzalimino-4,6-diamino-2(2 H)-pyridinone- 3-carbonitrile (15)}

Equimolecular amounts (0.01 mole) of 2 (1.87 g) and acetylacetone $(1 \mathrm{~g})$ were refluxed in dimethylformamide $(20 \mathrm{ml})$ for $2 \mathrm{~h}$. The reaction mixture was poured into ice/water acidified with few drops of hydrochloric acid. The solid product, so formed, was filtered off and crystallized from ethanol as buff crystals; m.p. $270{ }^{\circ} \mathrm{C}$; yield $1.88 \mathrm{~g}(75 \%)$. - IR $(\mathrm{KBr}$, $\left.\mathrm{cm}^{-1}\right): v(\mathrm{CN}): 2220 ; v(\mathrm{CO}): 1690$.

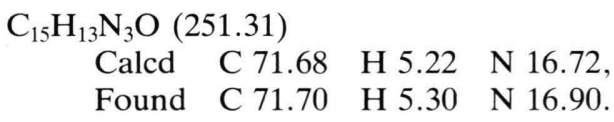

\section{Coupling of compound $\mathbf{1 4}$}

A solution of $\mathbf{1 4}(2.99 \mathrm{~g}, 0.01 \mathrm{~mole})$ in ethanol $(30 \mathrm{ml})$ containing sodium acetate $(3.0 \mathrm{~g})$ was coupled with an ice-cold solution of benzenediazonium chloride [prepared by adding sodium nitrite $(0.69 \mathrm{~g}$,
0.01 mole) to the appropriate quantity of aniline $(0.93 \mathrm{~g}, 0.01 \mathrm{~mole})$ in hydrochloric acid $(26 \mathrm{ml})]$. The solid product, so formed, was collected by filtration and crystallized from ethanol as red crystals; m.p. $195{ }^{\circ} \mathrm{C}$; yield $3.62 \mathrm{~g}(90 \%)$. - IR $\left(\mathrm{KBr}, \mathrm{cm}^{-1}\right)$ : $v(\mathrm{NH}): 3400,3300 ; v(\mathrm{CN}): 2200 ; v(\mathrm{CO}): 1710,1690$.

$\mathrm{C}_{22} \mathrm{H}_{21} \mathrm{~N}_{5} \mathrm{O}_{3}$ (403.48)

Calcd C 65.48 H 5.25 N 17.36,

Found C 65.60 H $5.30 \quad$ N 17.10.

Ethyl-(4-benzalhydrazido-5-methyl-2-phenyl-

$3(2 \mathrm{H})$-pyridazinimine)-6-carboxylate (18)

Compound 17 (4.03 g, 0.01 mole) was refluxed in a solution of acetic $(30 \mathrm{ml})$ - hydrochloric $(2 \mathrm{ml})$ acids mixture for $2 \mathrm{~h}$. The reaction product was poured into water. The solid obtained was collected by filtration and crystallized from acetic acid as brown crystals; m. p. $230{ }^{\circ} \mathrm{C}$; yield $3.02 \mathrm{~g}(75 \%)$. IR $\left(\mathrm{KBr}, \mathrm{cm}^{-1}\right): v(\mathrm{NH}): 3350,3200 ; v(\mathrm{CO}): 1720$, 1690

$$
\begin{aligned}
& \mathrm{C}_{22} \mathrm{H}_{21} \mathrm{~N}_{5} \mathrm{O}_{3}(403.48) \\
& \text { Calcd } \\
& \text { Found }
\end{aligned}
$$

Ethyl-(2-amino-4-methyl-3-benzalhydrazido6-trichloromethylpyridine)-5-carboxylate (20)

Trichloroacetonitrile $(1.44 \mathrm{~g}, 0.01 \mathrm{~mole})$ was added to a solution of $\mathbf{1 4}$ ( $2.99 \mathrm{~g}$; 0.01 mole) in refluxing dioxane $(30 \mathrm{ml})$ with a few drops of piperidine for $3 \mathrm{~h}$. The solid product obtained was collected by filtration and crystallized from ethanol as buff crystals; m.p. $220{ }^{\circ} \mathrm{C}$; yield $3.76 \mathrm{~g}(85 \%)$. - IR $(\mathrm{KBr}$, $\left.\mathrm{cm}^{-1}\right): v\left(\mathrm{NH}_{2}, \mathrm{NH}\right): 3450,3330 ; v(\mathrm{CO}): 1710,1680$.

$$
\begin{array}{cccc}
\mathrm{C}_{18} \mathrm{H}_{17} \mathrm{~N}_{4} \mathrm{O}_{3} \mathrm{Cl}_{3}(443.74) & & \\
\text { Calcd } & \mathrm{C} 48.71 & \mathrm{H} 3.86 & \mathrm{~N} 12.62, \\
\text { Found } & \mathrm{C} 48.60 & \mathrm{H} 3.80 & \mathrm{~N} 12.60 .
\end{array}
$$

[1] F. M. Abdelrazek, N. S. Ibrahim, Z. E. Kandeel, and M. H. Elnagdi, Synthesis 1984, 970.

[2] A. Habashi, N. S. Ibrahim, R. M. Mohareb, and S. M Fahmy, Liebigs Ann. Chem. 1986, 1632.

[3] K. Gewald, U. Hain, and M. Gruner, Chem. Ber. 1985, 2198.
[4] S. M. Fahmy, N. M. Abed, R. M. Mohareb, and M. H. Elnagdi, Synthesis 1982, 490.

[5] M. H. Elnagdi and H. Wamhoff, Chem. Lett. 1981, 419.

[6] J. L. Soto, C. Seoane, P. Zammorano, and F. Javier, Synthesis 1981, 529. 